\title{
A Research of Sensors Complementary for the RFID Wireless Network Based on the Variable Coverage Radius
}

\author{
Hongzhi Wang ${ }^{1,2, a}$, Yang Zhao ${ }^{3, \mathrm{~b}}$ and Haixia Zhou ${ }^{1, \mathrm{c}, *}$ \\ ${ }^{1}$ College of Economics and Management, Qingdao Agricultural University, \\ Qingdao, 266109, China \\ ${ }^{2}$ Postdoctoral Programme of China Industrial Economic Security Research \\ Center, Beijing Jiao tong University, Beijing 100044, China \\ ${ }^{3}$ School of Management, Shenyang Institute of Engineering, Shenyang, 110136, \\ China \\ ausagewhz@163.com, b35153051@qq.com, ${ }^{c}$ fun007-008@163.com \\ *Corresponding Author: Haixia Zhou
}

\begin{abstract}
In order to solve the problems of sensors complementary, algorithm sensors complementary for the RFID wireless network is proposed in this paper. Based upon the fundamental definitions about the probabilistic events and the data generated by the particle filtering algorithm, the paper considers the factors of the number of locations covered and the probability the object appears. In view of the definition of the covering variability and the cover ratio, 5kinds of RFID sensors covering radius are considered. Finally, the experiment is carried out in order to improve the algorithm's availability and effectiveness.
\end{abstract}

Keywords: RFID sensors; covering radius; RFID wireless sensors network; algorithm

\section{Introduction}

With the application of Radio Frequency Identification (RFID), because of considering the limitation of the scope which the sensor signal cover for the objects' tag, the cost of the sensor, the consuming of the energy and the efficiency of the work, the RFID wireless sensors site selection always are installed in the work region which the objects have the high frequency activity [1]. In order to fulfill the requirement of the design arrangement for the RFID system, the topic for the research of the RFID Network Planning Problem emerged and it can solve the problem of the design arrangement [2].

The previous research algorithms have randomness and do not consider the weight of the sensor. The randomness is caused by the random number in the evolution stages. If the individualities of the population are not interposed, the result produced by random cannot be the optimum solution for the projects.

Currently, because the difference of study objects and the conditions, the approaches of RFID wireless network site selection is only a reference for the actual projects. In the research of RFID Network Planning, Nandakumar Mysore and Ahmed Wasif Reza proposed the method that the whole workspace is filled with the area of RFID sensors when the number of the sensors is minimum, in order to cover the region using the RFID sensors signal and cost little $[3,4]$. For reducing the random, we need to analysis the distribution of the objects in the workspace. The usual method that estimates the distribution of the objects is particle filtering [5]. The particle filtering algorithm adopts the Bayes filtering and this method can produce the probabilistic distribution of the objects. 
In summary, the approach for RFID site selection almost needs the RFID data collected from reality as the reference, not considering the probability factors which the objects appear in the location. In the reality application, because of the all kinds of noise, the RFID date will contain many errors and the reality cover scope by sensors is not identical to the result by compute sometimes. The algorithm existed cannot solve these problem.

In order to solve the RFID Network Planning Problem, the paper proposed the definition of probabilistic timestamp event as the fundamental expression for cluster objects. The key factors considered in this method include the number of locations which the objects will appear and the probability. Based on the definition of cover density and the RFID sensors weight function, this paper presents an approach of sensors complementary for the RFID wireless network based on the variable covering radius. The users can analysis the RFID network through this approach to fill the area that cannot be covered. The users can get the scheme for the selection of the RFID sensors using the variable covering radius and this result can support the RFID network site optimization and analysis.

\section{Related Research Foundation Definition}

The basic events happen in the application of RFID means the action that the RFID readers fetch the object's tag [6] and every basic event happens at a time point.

A RFID basic event $e$ is expressed by the tuple $e=\left(\operatorname{Tag}, R_{v}\left(s_{A}\right)\right.$, Timestamp $)$. Tag represents the object's tag. $R_{v}\left(s_{A}\right)$ means the plane region which the RFID sensors sited in. the plane region is divides by Voronoi graph[7] and every region divided is represented by the point $s_{A}$, denoted by $R_{v}\left(s_{A}\right)$. timestamp represents the timestamp of the event.

The object may be several different locations at a timestamp and every location corresponds to its probability. So, before the definition of probabilistic timestamp events, we firstly introduce the definition of probabilistic events, as below:

Definition 1.( probabilistic events): a probabilistic event $e^{(t)}$ is composed by tuple( Tag $\left., R_{v}\left(s^{(t)}\right), t, P\right)$, Tag represents the object's tag, $R_{v}\left(s^{(t)}\right)$ represents the plane region which the object stay in. $t$ is the timestamp and $P$ is the probability which the object entrance the region $R_{v}\left(s^{(t)}\right)$ at timestamp $t$.

Definition 2.(probabilistic timestamp events): a probabilistic timestamp event $\bar{e}^{(t)}$ is composed by tuple( $\left.\operatorname{Tag}, t, \bigcup_{i=1}^{N}\left(R_{v}\left(s_{i}\right), P_{i}\right)\right)$, where $\sum_{i=1}^{N} P_{i}=1, N$ is the possible number of the locations which object appear. $R_{v}\left(s_{i}\right)$ represents the plane region which the object stay in., $t$ is the timestamp, $P_{i}$ is the probability which the object entrance the region $R_{v}\left(s_{i}\right)$.

Definition 3.(probabilistic centroid): Let a probabilistic timestamp event $\bar{e}^{(t)}$ contain $N$ regions $R_{v}\left(s_{i}\right), i=1 \ldots N$, the corresponding representing point's coordinate is $\left(x_{i}, y_{i}\right)$, probabilistic centroid is $c_{t}$, so

$$
c_{t}=\left(\sum_{i=1}^{N} x_{i} P_{i}, \sum_{i=1}^{N} y_{i} P_{i}\right) .
$$

Definition 4.(probabilistic timestamp events sequence): Let $\left[t_{i}, t_{j}\right]$ be the discrete timestamp interval, $t_{i} \leq t_{j}$. So the sequence $\left\{e^{\left(t_{i}\right)} \ldots \ldots e^{\left(t_{j}\right)}\right\}$ is the probabilistic 
timestamp events sequence for an object corresponding to discrete timestamp interval $\left[t_{i}, t_{j}\right]$.

According to definition 3, the probabilistic timestamp events sequence $e=\left\{e^{\left(t_{i}\right)}\right.$ $\left.\ldots . . e^{\left(t_{j}\right)}\right\}$ corresponds probabilistic timestamp centroid sequence $c=\left\{c_{t_{i}}, \ldots \ldots, c_{t_{j}}\right\}$.

The pattern of communication between the sensor and the receiver include Line of Sight (LOS) and Non Line of Sight (NLOS) [8]. Traditional approaches for location are generally based on LOS. RFID technology have the feature of untouchable and NLOS[9], so we consider these factors when decide the cover scope.

Definition 5.(cover density):Let $M$ be the number of sensors in the set $\mathfrak{R}$ in a RFID network, the cover radius of $\alpha_{i}$ is $r(\Re)$. Let $\bigcup e^{(t)}$ be the probabilistic timestamp events in the region whose central is $\alpha_{i}$ and radius is $r(\Re)$. Let $N_{i}$ be the possible number of locations and $\sum_{i=1}^{N} P_{i}$ the sum of the corresponding location probability. The cover density of sensor $\alpha_{i}$ is denoted by $D C\left(\alpha_{i}\right)$. $D C\left(\alpha_{i}\right)=\xi *\left(N_{i}\right)+$ $(1-\xi) * \sum_{i=1}^{N} P_{i}$,where $0<\xi<1$.

The parameter $\xi$ of definition 5 is the weight which involved the location and corresponding probability when we calculate the cover density. This parameter can be adjusted through the purpose of design.

Definition 6.(the minimum covering radius):Let $c^{\prime}$ be the covering clustering core of cluster $C$, for any objects $O \in C$, the mean of the distance between $c^{\prime}$ and $O$ is the minimum covering radius, denoted by

$$
\min _{-} r(C)=\frac{\left\|O-c^{\prime}\right\|}{|C|} \text {. }
$$

Definition 7.(the maximum covering radius):Let $c^{\prime}$ be the covering clustering core of cluster $C$, for any objects $O \in C$, the maximum of the distance between $c^{\prime}$ and $O$ is the maximum covering radius, denoted by $\max _{-} r(C)=\max \left\{\left\|O-c^{\prime}\right\|\right\}$.

The RFID covering radius of the sensors is during the interval [ $\min _{-} r(C)$, max_ $r(C)]$, so we introduce the definition of variable covering radius.

Definition 8.(the variable covering radius):Let $\min _{-} r(C)$ be the minimum covering radius of cluster $C, \max \_r(C)$ be the maximum covering radius. The variable covering radius

$$
\operatorname{var} r_{-} r=\min _{-} r(C)+\left(\max _{-} r(C)-\min _{-} r(C)\right) * \theta,
$$

where $\theta$ is the parameter of the variable covering radius.

The variable covering radius is acquired according to the parameter $\theta$ (as show in the Figure 1). We can set $\theta$ on the basis of the purpose.

In order to evaluate the effects of the difference covering radius, we introduce the definition of covering variability.

Definition 9.(the covering variability):Let $\min _{-} r$ be the minimum covering radius of cluster $C$, we use $r(C)$ as the covering radius, min_ $r$ correspond the cover density min_ $r\left(D C\left(\alpha_{i}\right)\right), r(C)$ correspond the cover density $r\left(D C\left(\alpha_{i}\right)\right)$. The covering variability of cluster $C$ for the covering radius $\min _{-} r$ and

$$
r(C) \text { is } \operatorname{dif}(C)=\left(r\left(D C\left(\alpha_{i}\right)\right)-\min _{-} r\left(D C\left(\alpha_{i}\right)\right)\right) / r\left(D C\left(\alpha_{i}\right)\right) .
$$




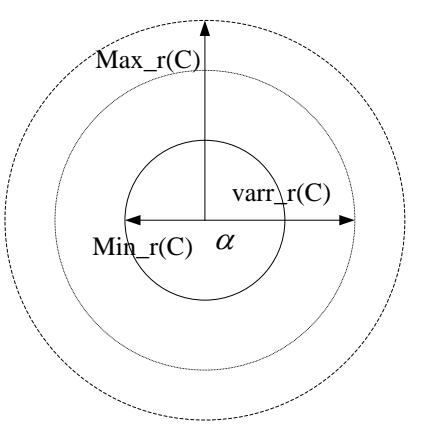

\section{Figure 1. The Minimum, the Maximum and the Variable Covering Radius}

The covering variability can be the parameter $\theta$ in the definition 8 . In this case, the variable radius is set according to the covering locations under the maximum covering radius and the minimum covering radius. It is the extending of radius base on the minimum covering radius so that it can cover more locations.

After the setting of the covering radius, for the effect evaluation of the complementary RFID sensors, we introduce the definition of the cover ratio for the RFID wireless sensors network.

Definition 10.(the cover ratio for the RFID wireless sensors network): Let $L$ the location set of the object, for any location $l_{i} \in L$, the responding probability is $p_{i}$ ,the location set covered by the sensors is Cover $\_$, the covering parameter is $\omega$, so the cover ratio for the RFID wireless sensors network is Cover_rate $=$ $\frac{\mid \text { Cover_L } L}{\mid L \omega+\sum p_{i} *(1-\omega)}$, where

$$
p_{i} \in \text { Cover_L } p_{i}^{\prime} \in L .
$$

\section{The Principle and Procedure of Algorithm}

Our approach contains two parts. The first part is an algorithm of sensors complementary for the RFID wireless network based on the covering cluster; the second part is the strategy for the selection of the RFID sensors' covering radius.

\subsection{The Method for the Selection of RFID Sensors}

Traditional cluster approach includes partitioning Clustering method, Hierarchical Clustering Method, Density clustering Method, Grid clustering Method and Model based clustering Method. The classical partitioning Clustering method is $\mathrm{K}$-kernel partitioning Clustering method which contains k-means algorithm and kmedoid algorithm.

The effect of the k-means algorithm is influenced by the initial Clustering cores greatly. The Traditional method for the initial Clustering cores is stochastic. If the selection of the cores is reasonable, the result will better and the velocity of clustering will faster. In order to disperse the initial Clustering cores, we need to give a constraint distance. So, in this paper, we give an approach of selection for the initial Clustering cores based on the self-adoptively. This approach means that the selection and the number of the initial Clustering cores are based on the clustering object's property, not stochastic.

Some of the RFID sensors' locations from the result of the covering clustering algorithm are not satisfied with the requirement. It needs to estimate the points which are accord with below restricted conditions: 
1. The number of the locations in the domain of the minimum covering radius is 1

2. The locations of the sensors which are added are in the domain of the original RFID network covering scope, in other words, the distance between them is less than $\mathrm{r}$;

3. The minimum covering radius min_ $r(C)=0$.

The approach of sensors complementary for the RFID wireless network based on the variable covering radius is as follow:

1) the division of the plane region

According to the method of Voronoi graph division, the RFID workspace will be divided into many sub regions and sign every region.

2) collect data and calculate the distribution

Particle filtering forecast the location of every particle in next timestamp. Then, resample the particles and select the particles which have the same location with RFID data that have the high probability. In order to calculate the distribution of the object, we in a nutshell use the ratio of particles quantity every location and the whole particles quantity.

According to the particle filtering algorithm, the output is the set of particle $\left\{x_{0: k}^{(i)}: i=1 \ldots N_{S}\right\}$, where $k$ the number of state is, $N_{S}$ is number of particle in $S$ state. It can express the posterior probability $p\left(x_{0: k} \mid z_{1: k}\right)$. where, $P\left(x_{k}=x_{k}^{i}\right)$ means the probability of that $x^{(i)}$ is at some locations. In other words, that is the distribution of the particle at state $k$.

3) collect the RFID data.

4) compute the locations of the RFID sensors added using the algorithm of sensors complementary for the RFID wireless network based on the variable covering radius.

Algorithm input: the collection of the RFID data; the data Marginal distribution; the initial number of cluster; the cover density parameter $\xi$; weight function parameter $\eta$; the cluster combine threshold $\mu$.

Algorithm output: The location of the RFID sensors added.

Procedure of Algorithm:

Step 1 Compute the object's location distribution without covered by the original RFID network.

According to the equipment or the collected RFID data, we compute the covering radius of the RFID sensors. Then, after eliminate the locations covered by the original RFID network, we get the remainder locations uncovered set $L$ _locations .

Step 2 For the elements of the set $L_{-}$locations, compute the sequence of the timestamp centroid.

According to the order of the timestamp, compute probabilistic centriod of every timestamp probabilistic event $\bar{e}^{(t)}$ to get the timestamp centroid sequence $C$.

Step 3 Compute the sequence of the covering density.

Let every centroid as the centrality, $r(\Re)$ as the covering radius. We choose $\xi$ as the covering density parameter to compute the covering density of every timestamp probabilistic event and reorder them by descending order to get the covering density sequence $C-S E Q$.

Step 4 Compute the cluster centrality sequence.

We choose the first centroid of $C-S E Q$ as the centrality of first cluster, denoted by $K_{1}$. In accordance with the sequence of $C-S E Q$, we choose the centroid from the beginning to the end with the constraint that the distances between centroids are 
greater than $2 * r(\Re)$. Let $K_{i}$ as the $i$ centroid and it satisfies $\left\|K_{i}-K_{j}\right\|>2 * r(\Re)$ ,where $K_{j}$ and $K_{i}$ are centroids, $1 \leq j \leq i$. In so doing, we get the clustering centroid sequence $K-S E Q$.

Let $N$ be the initial number of cluster, according to Step 4, the number of the centroid in sequence $K-S E Q$ is $M$. So, from the result, when $N>M$, the number of the centroids is $M$; when $N<M$, the former $N$ centroids as the clustering centrality.

Step 5 Cluster the particle marginal distribution data.

Scan the particle marginal distribution data by the order of timestamp. Let $E$ be the set of the particle marginal distribution data. Cluster $C_{i}$ corresponds the $i$ cluster centrality of sequence $K-S E Q$. For every probabilistic event $e^{(t)} \in E$, every initial cluster centrality $K_{i} \in K$, we compute $\min \left\{K_{i} \mid\left\|e^{(t)}-K_{i}\right\|\right\}$ to get $e^{(t)}$ to form $C_{i}$. According to the element of cluster $C_{i}$, we compute the cluster centrality again. Let $\left(x_{j}, y_{j}\right)$ be the coordinate of $e^{(t)}$, the coordinate of new cluster centrality $K_{i}^{\prime}$ is $\left(\sum_{i=1}^{N} x_{i} P_{i} / \sum_{i=1}^{N} P_{i}, \sum_{i=1}^{N} y_{i} P_{i} / \sum_{i=1}^{N} P_{i}\right)$. Then, we compute min_r $r\left(C_{i}\right)$. if min_ $r\left(C_{i}\right)=0$,go to Step 5 again, compute $i+1$ cluster centrality $K_{i+1}$;if min_r $r\left(C_{i}\right) \neq 0$,go to Step 6

Step 6 Let $K_{i}^{\prime}$ be the centrality. Let $\min \_r\left(C_{i}\right)$ be the radius. We form the circular covering region $K C_{i}$ and compute the centrality $K_{i}^{\prime \prime}$ of probabilistic event $e^{(t)}$ in $K C_{i}$, the coordinate is $\left(\sum_{i=1}^{N} x_{i} P_{i} / \sum_{i=1}^{N} P_{i}, \sum_{i=1}^{N} y_{i} P_{i} / \sum_{i=1}^{N} P_{i}\right)$.

Step 7 Compare $K_{i}^{\prime \prime}$ with $K_{i}^{\prime}$, if $K_{i}^{\prime \prime} \neq K_{i}^{\prime}$, go back to Step 5,else go to Step 8 .

Step 8 Compute the number of locations in the circular covering domain with the centrality is $K_{i}^{\prime \prime}$, min_ $r\left(C_{i}\right)$ is the radius. If the number is more than 1 , we add $K_{i}^{\prime \prime}$ into the final cluster centrality set $K F$. Inspect $K-S E Q$ to find whether there are remainder elements. If there are elements, go back to Step 5, else go to Step 9 。

Step 9 Inspect all the distance between the centralities in the set $K F$. If the distance is less than the cluster combine threshold $\mu$, we choose the mean value of the two centralities as the centrality of the combine cluster, go back to Step 6, else the algorithm end.

\subsection{The Strategy for the Choice of Covering Radius}

We have 5 methods for the radius choice:

1 . The minimum covering radius

This radius is generated from the above algorithm, which cover the locations with high probability. This radius is reference for other kind of radius.

2 . The maximum covering radius

We use the maximum covering radius of every cluster to completely cover. In this case, all the locations in the clusters will be completely covered. But the covering radius is the biggest one, the reality sensors' radius may can't reach that. At the same time, there will be a large scale of overlap with other sensors and may cause a lot of disturb.

3. The average covering radius

The mean value of the maximum radius and the minimum radius for every new added RFID sensor, the procedure is as follow: 
Step 1 compute min $r r\left(C_{i}\right)$ and $\max \_r\left(C_{i}\right)$ of sensor $\alpha_{i}$;

Step 2 compute sensor $\alpha_{i}$ 's average covering radius

$$
\text { ave_ } r\left(C_{i}\right)=\left(\min _{-} r\left(C_{i}\right)+\max _{-} r\left(C_{i}\right)\right) / 2 \text {; }
$$

4. The mean value of the average covering radius

In this case, we want to use the same radius for all sensors. So we consider the mean value of the average radius based on the average covering radius.

Step 1 for all $\alpha_{i} \in r(\Re)$, compute the mean value of the average radius

$$
\text { ave_ } r(\mathfrak{R})=\sum_{i=1}^{n} \text { ave_ } r\left(C_{i}\right) / N \text {, }
$$

where $N$ is the number of sensors;

Step 2 compute the covering variability $\operatorname{dif}\left(C_{i}\right)$ of all $\alpha_{i} \in r(\Re)$ for $\min _{-} r\left(C_{i}\right)$ and ave_ $r\left(C_{i}\right)$. If $\operatorname{dif}\left(C_{i}\right)=0$, it means that the amplified radius can't cover more objects. So, we can get the conclusion that this radius is inefficient.

5 . The variable covering radius

If the variability of the location number between the minimum covering radius and the maximum covering radius is remarkable, we consider using the variable covering radius.

Step 1 we get min_ $r\left(C_{i}\right)$ and max_ $r\left(C_{i}\right)$ of sensor $\alpha_{i}$ according to the above algorithm;

Step 2 compute the variability

$$
\operatorname{dif}(C)=\left(\max \_r\left(D C\left(\alpha_{i}\right)\right)-\min \_r\left(D C\left(\alpha_{i}\right)\right)\right) / \max \_r\left(D C\left(\alpha_{i}\right)\right) \text {; }
$$

Step 3 compute the variable covering radius $\left(\operatorname{var} r_{-} r\right)$ of sensor $\alpha_{i}$.

\subsection{Time Complexity of Algorithm}

Let $n$ be the number of cases in the collection of data of uncovered object' $\mathrm{s}$ RFID marginal distribution, $m$ be the number of RFID sensors added, $n_{i}$ be the number of marginal distribution for the object uncovered at every timestamp. The key procedure step3's time complexity is $O\left(\sum_{i=1}^{n} n_{i}\right)$. The key procedure Step4's time complexity is $O\left(\sum_{i=1}^{n} m_{i}\right)$. The key procedure step5 and step6's time complexity is $O(n \sqrt{n})$. So, we can get the whole algorithm's time complexity is

$$
O\left(\sum_{i=1}^{n} n_{i}+\sum_{i=1}^{m} n_{i} l_{j}+n \sqrt{n}\right) .
$$

\section{Experiment}

In order to test and verify our algorithm, we use the public data [10] from the Lahar project of the Washington University to make the Experiment.

\subsection{Experiment Data}

The Lahar project of the Washington University carried out a RFID ecosystem which is a very big project. This RFID ecosystem includes 150 RFID sensors which distribute around a 6 floors building. 
Table 1. The Collection of RFID Data(Partial)

\begin{tabular}{cc}
\hline timestamp & Sensor ID \\
\hline 1226636100426 & 103 \\
1226636101426 & 104 \\
1226636102426 & 104 \\
\hline
\end{tabular}

Table 2. Particle Filtering can Generate the Particle Marginal Distribution(Partial)

\begin{tabular}{cccc}
\hline Tag number & timestamp & location & probability \\
\hline 2254716205721250000 & 1226636093426 & 375787 & 0.887259257084265 \\
2254716205721250000 & 1226636093426 & 310450 & 0.083440545919008 \\
2254716205721250000 & 1226636093426 & 394154 & 0.029298595297040 \\
\hline
\end{tabular}

\subsection{The Selection of the RFID Sensors' Locations}

(1)According to the data from the Lahar project of the Washington university and the method of Voronoi graph, we divide the experiment region into sub regions, every region $R_{v}\left(s^{(t)}\right)$ denoted by a pointas show in Figure 2.

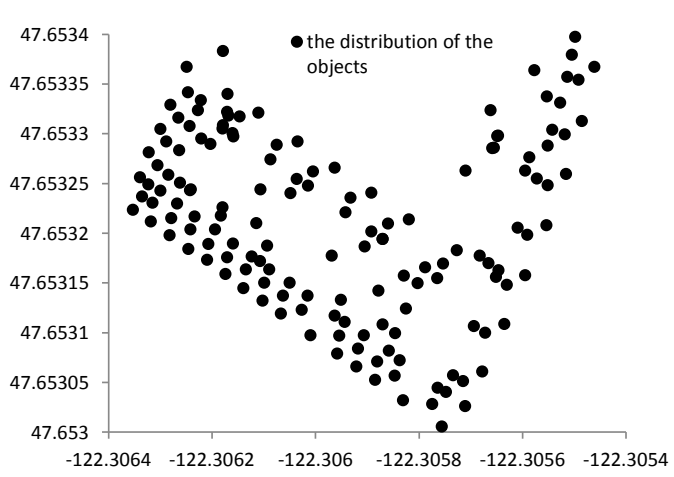

Figure 2. The Distribution of the Object's Location

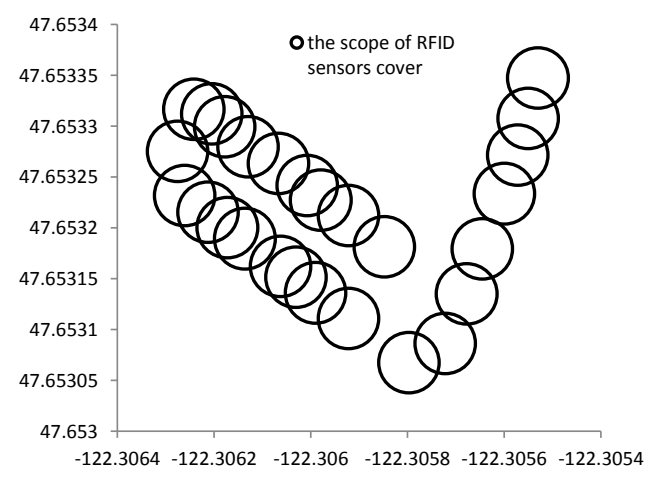

Figure 3. The covering scope of the initial RFID network

(2) the result of the algorithm of sensors complementary for the RFID wireless network based on the variable covering radius.

Firstly, Figure 2 shows the distribution of the objects in the workspace. According to Table 1 and Table 2, we get the existent RFID wireless network's covering scope as show in Figure 3. After elimination of the locations covered, we get the remainder distribution of object's locations uncovered as show in Figure 4According to the algorithm, we get the initial cluster's centrality and the regulatory centrality 's locations after adjusted as show in Figure 5. 


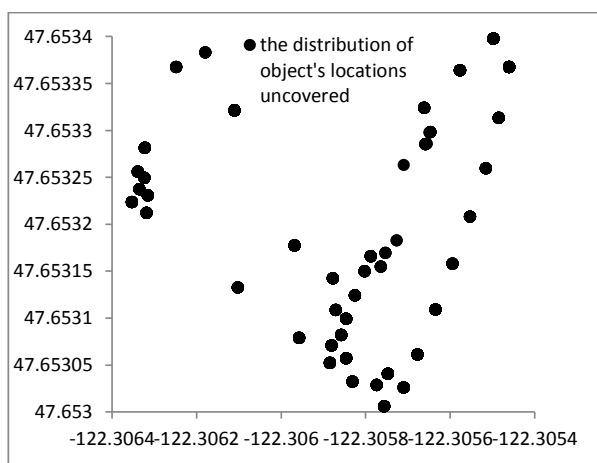

Figure 4. The Distribution of the Object's Locations Uncovered

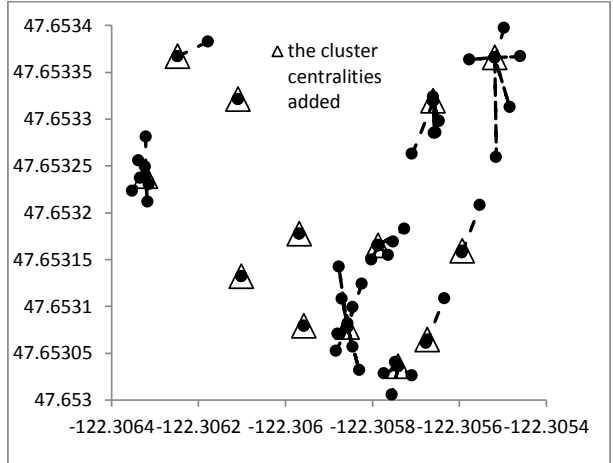

Figure 5. Initial Cluster Centralities

\subsection{The Selection of the Covering Radius of the RFID Sensors}

So, we can get the minimum covering radius $\min \_r(C)$ and the maximum covering radius max_ $r(C)$ as show in Figure 6.

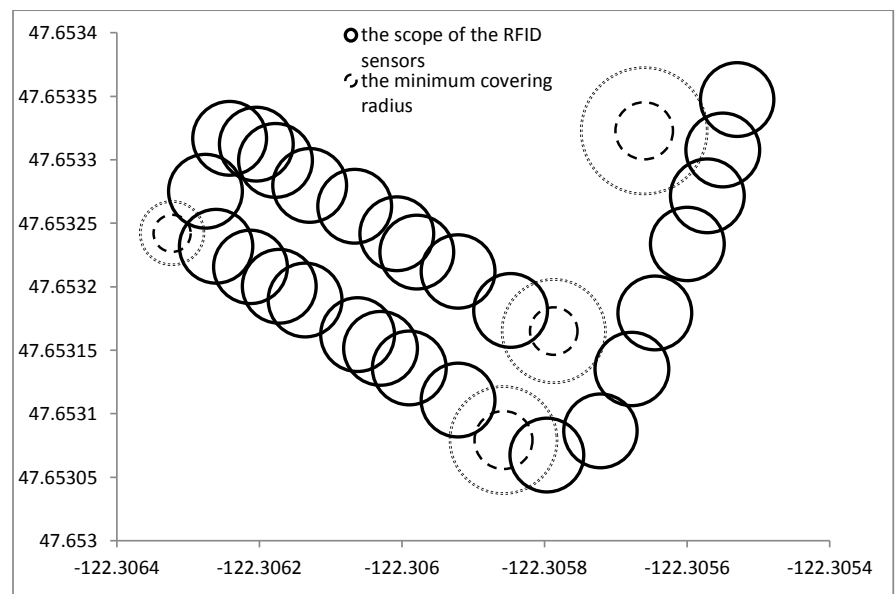

Figure 6. The Maximum Covering Radius and the Minimum Covering Radius of the Sensors Added

The analysis for the result of different covering radius: in this paper, we use the minimum covering radius as the reference and other covering radius as the comparison. The effect of different covering radius is as follow:

1. The maximum covering radius versus the minimum covering radius

The arrangement of the RFID sensors for the maximum covering radius is showed in Figure 7. From the result (as show in Figure 8), No.7 sensor both have the high covering density and the covering variability have a little change. It means the promotion of the covering efficiency is not remarkable. So, for this sensor, both of the radiuses are ok. The covering variability of the No.4 is very remarkable. It means, in this case, the maximum covering radius increase the number of the object's locations. The covering density of No.0 and No.11 sensors both are very little and the covering variability is not remarkable. So, the adjustment of this sensor is inefficiency. 


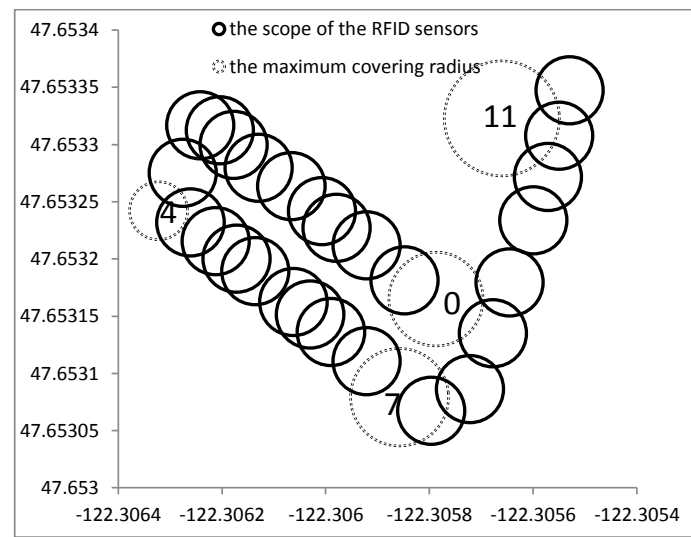

Figure 7. The Added Sensors of the Maximum Covering Radius

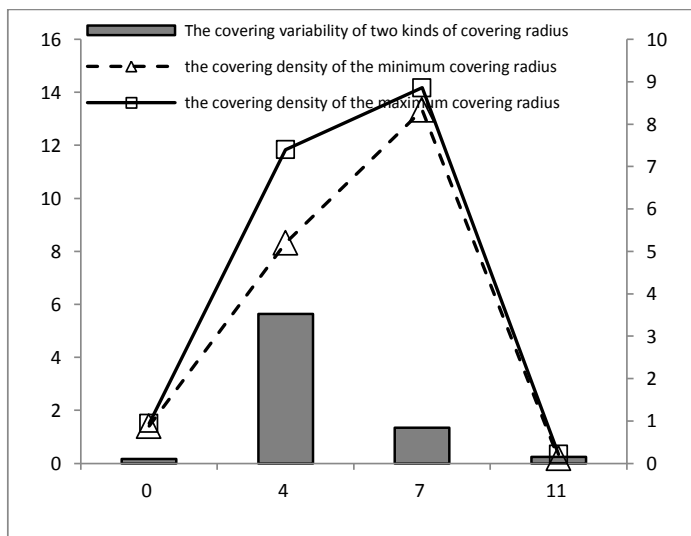

Figure 8. The Effect of Sensors Added

2. The average covering radius versus the minimum covering radius

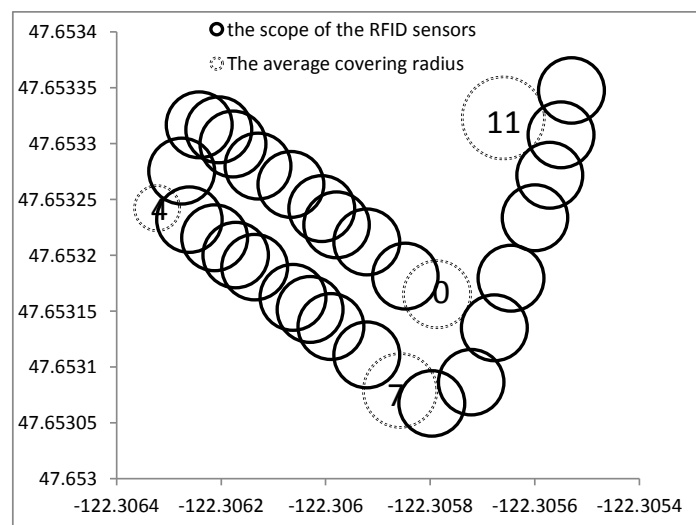

Figure 9. The Added Sensors of the Average Covering Radius 


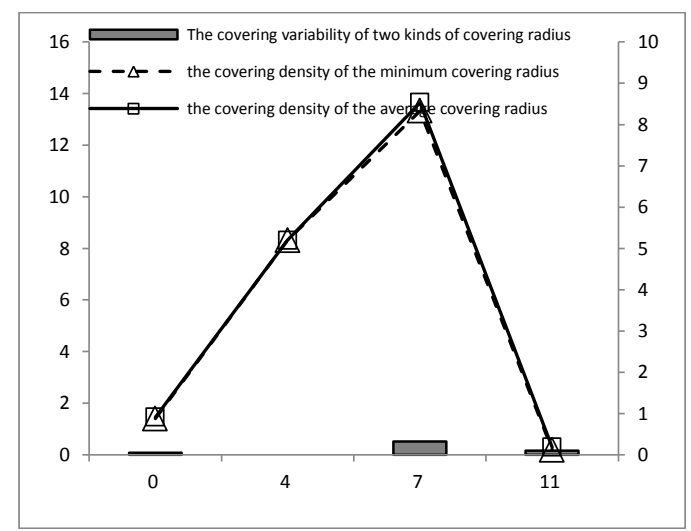

Figure 10. The Effect of Sensors Added

The arrangement of the RFID sensors for the average covering radius is showed in Figure 9. From the result (as show in Figure 10), the sensors of No.0, No.7, No.11 all have very little covering variability. In this case, the average covering radius can't promote the covering efficiency.

3 . The mean value of the average covering radius versus the minimum covering radius

The arrangement of the RFID sensors for The mean value of the average covering radius is showed in Figure 11. From the result (as show in Figure 12, No.7 sensor both have the high covering density and the covering variability have a little change. It means the promotion of the covering efficiency is not remarkable. So, for this sensor, both of the radiuses are ok. The covering variability of the No.4 is very remarkable. It means, in this case, the mean value of the average covering radius increase the number of the object's locations. The covering density of No.0 and No.11 sensors both are very little and the covering variability is not remarkable. So, the adjustment of this sensor is inefficiency.

4. The variable covering radius versus the minimum covering radius

The arrangement of the RFID sensors for the variable covering radius is showed in Figure 13. From the result (as show in Figure 14), all the sensors have very little covering variability. So, In this case, the average covering radius can't promote the covering efficiency.

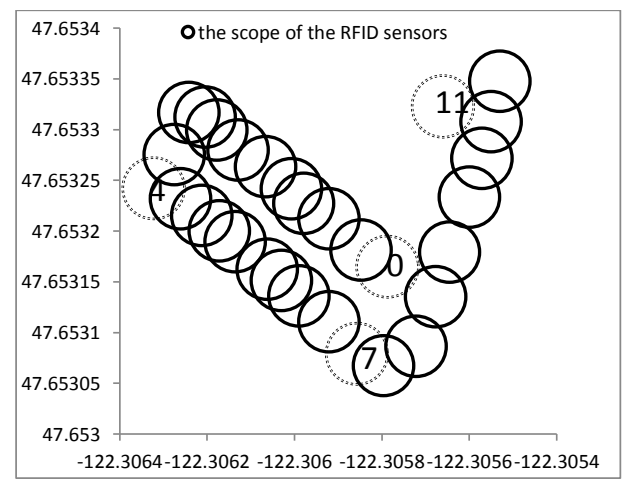

Figure 11. The Added Sensors of the Average Covering Radius 


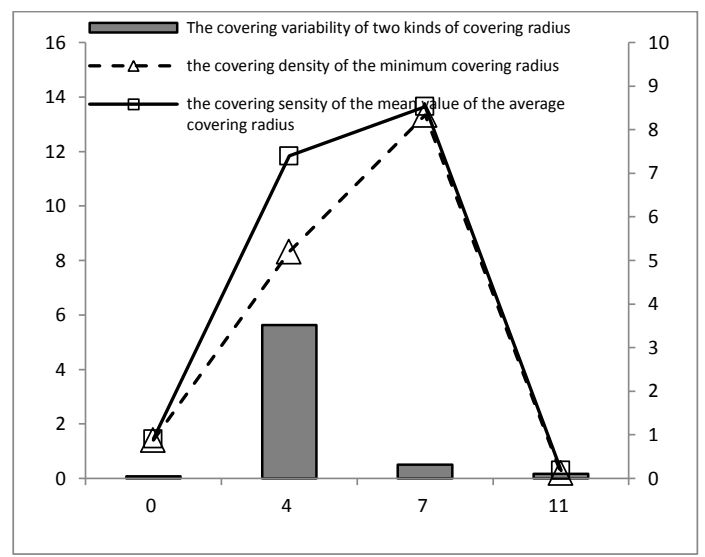

Figure 12. The Effect of Sensors Added

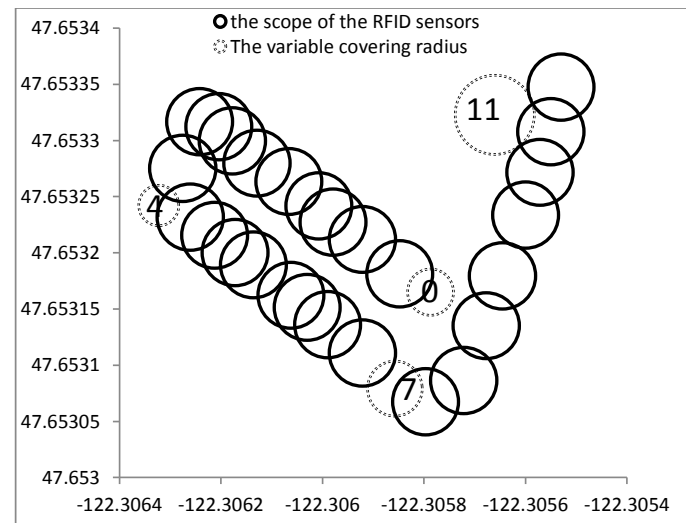

Figure 13. The Added Sensors of Variable Covering Radius

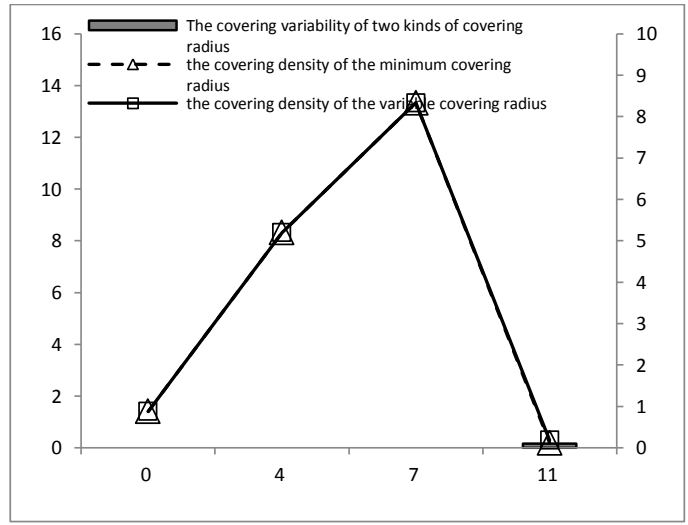

Figure 14. the Effect of Sensors Added

\section{Summary}

In this paper, in order to solve the problem of the sensors complementary for RFID wireless network, we propose an approach of sensors complementary for the RFID wireless network based on the variable covering radius. We use this method to make the analysis for the RFID networks to achieve the locations uncovered by sensors as the foundations of complementary method. The definition of cover density and the covering ratio consider the factors which include the number of the locations covered by sensors and the probability of object appear in this location. 


\section{Acknowledgments}

The authors gratefully acknowledge the support of Advanced Talents Foundation of QAU Nos. 6631115710, Shandong Province Social Science Project Nos.12BJJJ06 and Humanities and Social Sciences Research Foundation of QAU (Nos. 6611115726, Nos.614Y02).

\section{References}

[1] J. Waldrop, D. W. Engels and S. E. Sarma, "A MAC for RIFD reader networks", IEEE Wireless Communications and Networking (WCNC), vol. 3, (2003), pp. 1701-1704.

[2] H. Chen and Y. Zhu, "RFID networks planning using evolutionary algorithms and swarm intelligence", In: 4th International Conference on Wireless Communications, Networking and Mobile Computing (WiCOM)), (2008), pp. 1-4.

[3] N. Mysore, P. Nenavat and R. S. Mulukutla, "An efficient algorithm for rfid reader positioning for coverage of irregularly shaped areas", 5th Annual IEEE Conference on Automation Science and Engineering Bangalore, India, (2009), pp. 233-240.

[4] A. W. Reza and T. K. Geok, "Investigation of indoor location sensing via RFID reader network utilizing grid covering algorithm", Wireless Pers. Commun., vol. 49, (2009), pp. 67-80.

[5] C. Dechang, W. Jiejun and W. Qinglin, "RFID indoor nodes tracking based on particle filtering technology", Information Technology, no. 08, (2012).

[6] F. Wang, S. Liu and P. Liu, "Complex RFID event processing, The VLDB Journal", vol. 18, (2009), pp. 913-931.

[7] Z. Wenbin and Z. Zhengxu, "Research on Engineering Data Format Conversion Based on Voronoi Diagram", Computer Engineering, vol. 38, no. 6, (2012).

[8] J. Wang, "Toward Robust Indoor Localization Based on Bayesian Filter Using Chirp-Spread-Spectrum Ranging”, IEEE Transactions On Industrial Electronics, vol. 59, no. 3, (2012).

[9] B. Hameed, "Self-calibration of RFID Reader Probabilities in a Smart Real-Time Factory", J. Kay et al. (Eds.): Pervasive 2012, LNCS 7319, (2012), pp. 253-270.

[10] http://lahar.cs.washington.edu/displayPage.php?path=//content/Download/RFIDData/rfidData.html.

[11] H. Hanpang and C. Yingting, "Robust design for rfid system testing and applications", 2007 IEEE International Conference on Robotics and Automation. Roma, Italy, vol. 4, (2007), pp. 594-4 597.

[12] S. Anusha, "A coverage planning tool for RFID networks with mobile readers", Bombay: Indian Institute of Technology Bombay, (2005).

[13] S. H. Siadat and A. Seamat, "Location based system for mobile devices using RFID", Senond Asia International Conference on Modelling \& Simulation, (2008), pp. 291-294.

[14] L. Li-fang and Z. Ming-zheng, "A Clustering Algorithm Based on Constructive Kernel Covering Algorithm, Computer Technology and Development, vol. 19, no.1. (2009). 
International Journal of Future Generation Communication and Networking Vol. 9, No. 12 (2016) 